\title{
Consumo de alcohol, tabaco y otras drogas en chicas en edad escolar: relación con la edad y los niveles de actividad física
}

Consumo de álcool, tabaco e outras drogas em meninas em idade escolar: relação com a idade e os níveis de atividade física

\author{
M. Bragança $a^{*} ;$ S. Márquez ${ }^{2}$; A. Salguero ${ }^{2}$ \\ ${ }^{I}$ Departamento de Educação Física, Universidade Federal de Sergipe, 49100-000, São Cristóvão-SE, Brasil \\ ${ }^{2}$ Departamento de Educación Física y Deportiva, Instituto de Biomedicina (IBIOMED), Universidad de León, 24071, \\ León, España
}

*email: marthabragancaufs@gmail.com

(Recebido em 22 de outubro de 2015; aceito em 17 de novembro de 2015)

\begin{abstract}
Adoptar un estilo de vida activo y saludable ayuda en la prevención de distintas enfermedades y comportamientos de riesgo. El objetivo de este estudio fue identificar el nivel de actividad física (NAF) y verificar su influencia sobre la edad y el consumo de drogas lícitas e ilícitas, en escolares del sexo femenino. Participaron 572 chicas con edad entre 11 y 18 años (14,6 2 , 1años) de colegios de la ciudad de León-España. Para la valoración del NAF se utilizó la versión española (Cuestionario de Valoración de la Actividad Física) del Four-by-One-Day Physical Activity Questionnaire. Para los hábitos de consumo se utilizó el Inventario de Conductas de Salud en Escolares. Un porcentaje importante de escolares $(68,2 \%)$ eran inactivas. Al analizar separadamente los días entre semana y el fin de semana, dicho porcentaje era de un $50,1 \%$ y un $82,1 \%$, respectivamente. Independientemente del período de la semana, la actividad física se reducía con la edad y aumentaba el consumo de todos los tipos de drogas $(\mathrm{p}=0,001)$. El grado de actividad física se relacionó significativamente con la edad $\left(\chi^{2}=25,109, \mathrm{p}=0,003\right)$ y con el consumo de alcohol $\left(\chi^{2}=12,993, \mathrm{p}=0,005\right)$, no así con el de tabaco $\left(\chi^{2}=6,953, \quad \mathrm{p}=0,073\right)$, ni con el consumo de drogas ilícitas $\left(\chi^{2}=2,145, p=0,54\right)$. En conclusión, los datos del presente estudio indican la existencia de una relación inversa del nivel de actividad física de escolares del sexo femenino con la edad y el consumo del alcohol.
\end{abstract}

Palabras-chave: Actividad motora, Drogas ilícitas, Adolescentes.

Adotar um estilo de vida ativo e saudável ajuda na prevenção de distintas doenças e comportamentos de risco. O objetivo deste estudo foi identificar o nível de atividade física (NAF) e verificar sua influência sobre a idade e o consumo de drogas lícitas e ilícitas em estudantes do sexo feminino. Participaram 572 meninas com idades entre 11 e 18 anos (14,6 $\pm 2,1$ anos) de escolas da cidade de León-Espanha. Para a avaliação do NAF se utilizou a versão espanhola (Cuestionario de Valoración de la Actividad Física) do Four-by-One-Day Physical Activity Questionnaire. Para os hábitos de consumo se utilizou o Inventario de Condutas de Saúde em Escolares. Uma porcentagem importante de estudantes $(68,2 \%)$ era inativa. Ao analisar separadamente os dias entre semana e o fim de semana, esta porcentagem era de um 50,1\% e um $82,1 \%$, respectivamente. Independentemente do período da semana, a atividade física diminuía com a idade e aumentava o consumo de todos os tipos de drogas $(\mathrm{p}=0,001)$. O nível de atividade física se relacionou significativamente com a idade $\left(\chi^{2}=25,109, \mathrm{p}=0,003\right)$ y com o consumo de álcool $\left(\chi^{2}=12,993\right.$; $\mathrm{p}=0,005)$, mas não com o de tabaco $\left(\chi^{2}=6,953 ; \mathrm{p}=0,073\right)$, nem com o consumo de drogas ilícitas $\left(\chi^{2}=2,145 ; \mathrm{p}=0,54\right)$. Em conclusão, os dados do presente estudo indicam a existência de uma relação inversa do nível de atividade física de estudantes do sexo feminino com a idade e o consumo de álcool.

Palavras chave: Atividade motora, Drogas ilícitas, Adolescentes.

\section{INTRODUCIÓN}

Diversos estudios señalan que adoptar un estilo de vida activo y saludable ayuda en la prevención de distintas enfermedades y comportamientos de riesgo [1]. No obstante, para todas las franjas de edad el sedentarismo, el uso de nuevas tecnologías y el consumo de drogas van 
creciendo en todos los países y son señalados como un factor de riesgo importante para el desarrollo de enfermedades crónico degenerativas, cardiovasculares, obesidad y diabetes [2,3].

Está sobradamente probado que la adolescencia es un período clave en la adquisición de una serie de conductas que pueden determinar el estilo de vida de un sujeto. Durante este período, la orientación de los hábitos puede dirigirse hacia un patrón de vida saludable o, por el contrario, hacia un patrón en el que primen conductas perjudiciales para la salud. Debido a la importancia de este tema, desde hace décadas se han desarrollado multitud de estudios en torno al mismo [4].

Los estudios relacionados con estilos de vida en los adolescentes apuntan una disminución de la práctica de actividad física, lo que conlleva a un estilo de vida sedentario, siendo las adolescentes del sexo femenino las que presentan un mayor índice de inactividad respecto a los del sexo masculino $[3,5,6]$.

En definitiva, adoptar un estilo de vida activo y sano, sin consumo de drogas lícitas e ilícitas, afecta la calidad de vida de los sujetos. Así, el objetivo de este estudio fue identificar los niveles de actividad física y verificar su influencia sobre la edad e indicadores del estilo de vida como el consumo de drogas lícitas (alcohol y tabaco) y de drogas ilícitas, en escolares del sexo femenino.

\section{MATERIAL Y MÉTODOS}

Este es un estudio no experimental, transversal, descriptivo, con un abordaje cuantitativo [7], en el que participaron un total de 572 escolares del sexo femenino, con edades que variaban entre 11 y 18 años (14,6 $\pm 2,1$ años), de los cursos de $6^{\circ}$ Primaria, Educación Secundaria Obligatoria (E.S.O) y Bachillerato, de colegios de la ciudad de León-España.

Los datos recogidos fueron peso, altura, gasto calórico, edad, consumo de alcohol, tabaco y drogas ilícitas. Para la toma de datos respecto al peso se utilizó una báscula calibrada Seca $703^{\circledR}$, (Hamburgo, Alemania) y para la altura un estadiómetro Detecto D52 ${ }^{\circledR}$ (Webb City, MO, Estados Unidos).

La valoración del NAF se llevó a cabo mediante la versión española (Cuestionario de Valoración de la Actividad Física) [8] del cuestionario Four-by-One-Day Physical Activity Questionnaire (FBODPAQ) [9], en sus dos formas - Jornada Escolar y Fin de Semana. El Cuestionario Jornada Escolar valora los hábitos diarios en una jornada escolar, es decir, de lunes a viernes (antes de la escuela, durante las clases, recreos, hora de la comida, y después de la escuela). El Cuestionario Fin de Semana evalúa hábitos diarios en una jornada de fin de semana (por la mañana, tarde y noche). Ambos registran todo tipo de actividad física (deportiva, cotidiana o laboral). Por medio de esos datos se pudo estimar el gasto calórico total expresado en kcal. $\mathrm{kg}^{-1} \cdot \mathrm{dia}^{-1}$ en cada uno de esos periodos multiplicando el tiempo invertido en cada actividad por sus respectivos mets. Así se pudo clasificar las escolares en cuatro NAF: 1- muy inactivo $\left(<33,0 \mathrm{kcal} \cdot \mathrm{kg}^{-1} \cdot \mathrm{dia}^{-1}\right) ; 2$ - inactivo $\left(33,0\right.$ a $\left.36,9 \mathrm{kcal} \cdot \mathrm{kg}^{-1} \cdot \mathrm{dia}^{-1}\right) ; 3$ - moderadamente activo (37,0 a 39,9 kcal.kg $\left.{ }^{-1} \cdot \mathrm{dia}^{-1}\right)$; y 4 - activo $\left(\geq 40,0 \mathrm{kcal} \cdot \mathrm{kg}^{-1} \cdot \mathrm{dia}^{-1}\right)$ [8]. Los análisis del NAF fueron efectuados tomándose los valores de la semana completa (SC) así como de la jornada escolar (JE) y fin de semana (FS) por separado.

Para obtener los datos sobre los hábitos de consumo respecto a drogas lícitas e ilícitas, se utilizó la versión española [10] del cuestionario Inventario de Conductas de Salud en Escolares (The Health Behavior in School-Aged Children (1985/86): A WHO cross-national-survey HBSC) [11]. Dicho cuestionario fue elaborado por la Oficina Regional Europea de la Organización Mundial de la Salud (OMS) para el desarrollo de su programa de investigación internacional sobre los estilos de vida de los adolescentes y es el cuestionario específico para adolescentes que recoge un mayor número de variables sobre el estilo de vida.

Se informó previamente a la dirección de los centros escolares participantes, alumnas y sus respectivos padres o responsables, acerca de los objetivos y procedimientos del estudio. La participación fue voluntaria y antes de la aplicación de los cuestionarios se recogieron los consentimientos informados y firmados por los padres o tutores en el caso de las menores de edad. Se aplicaron los cuestionarios de acuerdo a los preceptos éticos en la investigación con 
seres humanos. Dicha aplicación garantizó el anonimato y la confidencialidad, y los sujetos contaron con la ayuda del equipo de investigadores. El protocolo del estudio fue aprobado por el Comité Ético de la Universidad de León (2007/00066/001).

Para la caracterización de la muestra se utilizó la estadística descriptiva (media y desviación estándar). El tratamiento inferencial se verificó mediante Chi-Cuadrado. El nivel de significación adoptado fue $\mathrm{p} \leq 0,05$.

Los análisis se llevaron a cabo utilizando el paquete estadístico para las Ciencias Sociales SPSS (Chicago, Estados Unidos), versión 21,0 para Windows.

\section{RESULTADOS Y DISCUSIÓN}

Las escolares tenían una edad entre 11 y 18 años, con una media de $14,6( \pm 2,1)$ años, y fueron divididas en cuatro grupos de edad, con in intervalo de dos años (Tabla 1).

Tabla 1: Distribución de las escolares por grupos de edad en años $(N=572)$

\begin{tabular}{ccc}
\hline Edad (años) & $\%$ & $\mathrm{n}$ \\
\hline $11-12$ & 29,2 & 167 \\
$13-14$ & 24,8 & 142 \\
$15-16$ & 31,1 & 178 \\
$17-18$ & 14,9 & 85 \\
\hline
\end{tabular}

Para verificar la influencia del NAF sobre las variables estudiadas, inicialmente habría que identificar los niveles de las escolares. De esta manera, fueron clasificadas en cuatro NAF de acuerdo con el gasto calórico, como queda reflejado en la Tabla 2.

Tabla 2: Nivel de actividad física de las escolares en la semana completa, jornada escolar y en el fin de semana $(N=572)$

\begin{tabular}{lccc}
\hline \multicolumn{1}{c}{ NAF } & $\begin{array}{c}\text { Semana completa } \\
\%(\mathrm{n})\end{array}$ & $\begin{array}{c}\text { Jornada escolar } \\
\%(\mathrm{n})\end{array}$ & $\begin{array}{c}\text { Fin de semana } \\
\%(\mathrm{n})\end{array}$ \\
\hline Muy inactivo & $43,7(250)$ & $31,5(180)$ & $67,1(384)$ \\
Inactivo & $24,5(140)$ & $18,6(107)$ & $15,0(86)$ \\
Moderadamente activo & $11,9(68)$ & $10,0(57)$ & $6,1(35)$ \\
Activo & $19,9(114)$ & $39,9(228)$ & $11,8(67)$ \\
\hline
\end{tabular}

Observando la Tabla 2, se pone de manifiesto que las escolares eran inactivas en un 68,2\% cuando se consideraba la semana completa. Al analizar separadamente los días entre semana (lunes a viernes, JE) las escolares inactivas suponían un 50,1\%, aumentando el porcentaje de aquellas activas hasta un 39,9\%. Durante el FS, los datos mostraron que las escolares se distribuyeron predominantemente en las categorías inactivas $(82,1 \%)$.

Estudios previos de Béltran-Carrillo, Devís-Devís, y Peiró-Velertet (2012) [12] y Farias Júnior et al., (2009) [2], analizando los datos semanales de manera global, encontraron una predominancia de los NAF muy inactivo e inactivo en la SC, muy similar a lo que ocurrió en al presente estudio. Por otro lado, al analizar de forma separada los dos periodos, el hecho de que las escolares se presentasen más inactivas en el FS se asemeja a datos de otros estudios desarrollados en distintos contextos españoles y extranjeros [13, 14, 15, 16]. No obstante, trabajos de Cantera-Garde, y Devís-Devís (2000) [17], Molinero, Martinez, Garatachea, y Márquez (2010) [18], y Peiró-Velert, Devís-Devís, Beltrán-Carrillo, y Fox (2008) [19], apuntan a que las escolares fueron más activas en el FS.

Tal vez esas diferencias sean debidas a la utilización del tiempo libre. En la JE no existe mucha alternativa para inclusión de actividades en el tiempo libre, una vez que los horarios y tareas están estructurados para atender, principalmente, a las necesidades de la escuela, mientras que en el FS podría existir mayor disponibilidad para la utilización de ese tiempo con distintas opciones de elección de actividades. En todo caso, se puede afirmar que existe una influencia del día de la semana sobre el NAF de escolares. 
A continuación se compararon los NAF en relación a los grupos de edad, mostrándose los resultados en la Tabla 3.

Tabla 3: Nivel de actividad física de las escolares en la semana completa, jornada escolar y en el fin de semana en relación a los grupos de edad (años) (N=572)

\begin{tabular}{|c|c|c|c|c|c|}
\hline \multirow[b]{2}{*}{ NAF } & \multicolumn{4}{|c|}{ Grupos de edad (anos) } & \multirow[b]{2}{*}{$\mathrm{p}$} \\
\hline & $\begin{array}{c}11-12 \\
\mathrm{n}=167 \\
\%(\mathrm{n})\end{array}$ & $\begin{array}{c}13-14 \\
\mathrm{n}=142 \\
\%(\mathrm{n})\end{array}$ & $\begin{array}{c}15-16 \\
\mathrm{n}=178 \\
\%(\mathrm{n})\end{array}$ & $\begin{array}{c}17-18 \\
\mathrm{n}=85 \\
\%(\mathrm{n})\end{array}$ & \\
\hline & \multicolumn{4}{|c|}{ Semana completa } & \multirow{5}{*}{0,003} \\
\hline Muy inactivo & $31,7(53)$ & $43,7(62)$ & $52,2(93)$ & $49,4(42)$ & \\
\hline Inactivo & $27,5(46)$ & $19,0(27)$ & $23,6(42)$ & $29,4(25)$ & \\
\hline Moderadamente activo & $16,8(28)$ & 13,4 (19) & $7,9(14)$ & $8,3(07)$ & \\
\hline Activo & $24,0(40)$ & $23,9(34)$ & $16,3(29)$ & $12,9(11)$ & \\
\hline \multicolumn{5}{|c|}{ Jornada escolar } & \multirow{5}{*}{0,001} \\
\hline Muy inactivo & $25,7(43)$ & $28,9(41)$ & $33,1(59)$ & $43,5(37)$ & \\
\hline Inactivo & $16,2(27)$ & $16,9(24)$ & $24,2(43)$ & $15,3(13)$ & \\
\hline Moderadamente activo & $9,0(15)$ & $9,2(13)$ & $6,7(12)$ & $20,0(17)$ & \\
\hline \multirow[t]{2}{*}{ Activo } & $49,1(82)$ & $45,0(64)$ & $36,0(64)$ & $21,2(18)$ & \\
\hline & \multicolumn{4}{|c|}{ Fin de semana } & \multirow{5}{*}{0,116} \\
\hline Muy inactivo & $63,5(106)$ & $65,5(93)$ & $73,6(131)$ & $63,5(54)$ & \\
\hline Inactivo & $13,2(22)$ & $14,8(21)$ & $14,1(25)$ & $21,2(18)$ & \\
\hline Moderadamente activo & $9,0(15)$ & $5,6(08)$ & $5,6(10)$ & $2,4(02)$ & \\
\hline Activo & $14,3(24)$ & $14,1(20)$ & $6,7(12)$ & $12,9(11)$ & \\
\hline
\end{tabular}

Los datos apuntan que, independiente del período de la semana, fueron las más jóvenes las más activas. También se observa que a medida que la edad evolucionó, las escolares se volvieron significativamente menos activas. En la SC asumir determinado NAF dependió, significativamente, de la edad $\left(\chi^{2}=25,109 ; \mathrm{p}=0,003\right)$. Analizándose separadamente los dos períodos, en la JE el NAF también tuvo una relación significativa con la edad $\left(\chi^{2}=33,679\right.$; $\mathrm{p}=0,001)$. Parece que ser más joven supone una mayor posibilidad de ser más activa. En cambio, en el FS, ser más o menos inactiva, no mostró una asociación significativa con la edad $\left(\chi^{2}=14,190 ; p=0,116\right)$. Independientemente de la edad, las escolares tuvieron un elevado grado de inactividad en el FS.

Las actividades practicadas por las más jóvenes en la escuela parecen determinar la existencia de un NAF más elevado en la JE respecto a las más mayores. Tal hecho puede indicar que la escuela ofrece actividades que facilitan el desarrollo de NAF más activos.

Cuando se analiza el NAF en relación a las edades, varios estudios han puesto de manifiesto que se produce una reducción en los niveles al aumentar la edad, coincidiendo con los resultados de la presente investigación $[2,3,5,12,13,14,18,20,21]$, aunque en algún caso no se ha confirmado dicha tendencia [17].

Independientemente de la coincidencia de los hallazgos, parece que los patrones de actividad física se alteran significativamente con el crecimiento. Los NAF sufren una disminución de la infancia hacia la adolescencia y siguen disminuyendo con posterioridad. Cuando se tiene una edad mayor ocurre una necesidad de invertir más tiempo en los estudios, ya se alcanzó una independencia personal, y ocurren cambios sociales respecto a salir más con amigos, ir al cine, fiestas, viajes. Estos factores pueden contribuir al aumento de la obesidad, de enfermedades degenerativas y de otras no transmisibles en la población [20].

En definitiva, las escolares fueron más activas en la JE que en el FS y, respecto a la edad, tal comportamiento predominó entre las más jóvenes. No obstante, es importante resaltar el grado de inactividad, en todas las franjas de edad, en el FS.

Para los análisis del NAF de las escolares en relación al estilo de vida respecto al consumo de sustancias adictivas, se consideraron los valores de la semana completa, agrupando todas las actividades, independientemente de si eran físicas, deportivas, competitivas, laborales, 
individuales o colectivas, reflejando al final, un único grado de actividad física clasificado dentro de sus respectivas categorías. Inicialmente se analizó la relación entre el consumo de drogas licitas e ilícitas y los grupos de edad, cuyos datos están reflejados en la Tabla 4.

Tabla 4: Consumo de drogas lícitas (tabaco y alcohol) y drogas ilícitas respecto a los grupos de edad de las escolares $(N=572)$

\begin{tabular}{|c|c|c|c|c|c|c|c|}
\hline \multirow{2}{*}{\multicolumn{3}{|c|}{ Consumo \% (n) }} & \multicolumn{4}{|c|}{ Grupos de edad (años) } & \multirow{3}{*}{$\mathrm{p}$} \\
\hline & & & $11-12$ & $13-14$ & $15-16$ & & \\
\hline \multirow{3}{*}{ Alcohol } & & & & & & & \\
\hline & Sí & & $19,8(33$ & $61,3(87)$ & $91,0(162)$ & $98,8(84)$ & \multirow{2}{*}{0,001} \\
\hline & No & & $80,2(134)$ & 38,7 & $9,0($ & $1,2(01)$ & \\
\hline \multirow{2}{*}{ Tabaco } & Sí & & 6,0 & $27,5(39)$ & $43,3(77)$ & $70,6(60)$ & \multirow{2}{*}{0,001} \\
\hline & No & 67,5 & $94,0(157)$ & $72,5(103)$ & $56,7(101)$ & $29,4(25)$ & \\
\hline \multirow{2}{*}{$\begin{array}{l}\text { Drogas } \\
\text { ilícitas }\end{array}$} & Sí & $21,5(12$ & $0,6(01)$ & $11,3(16)$ & $28,1(50)$ & $65,9(56)$ & \multirow{2}{*}{0,001} \\
\hline & No & $78,5(449)$ & $99,4(166)$ & $88,7(126)$ & $71,9(128)$ & $34,1(29)$ & \\
\hline
\end{tabular}

Los datos registrados señalan que el consumo de todos los tipos de drogas se relacionaba significativamente con la edad $(\mathrm{p}=0,001)$. A medida que esta avanzó, ocurrió una alteración significativa en el patrón de consumo en todas las franjas de edad. Las escolares más mayores fueron las que más consumieron, tanto drogas lícitas, como ilícitas. Parece que, el hecho de ser más mayor, induce un consumo más elevado de drogas. Otro punto a observar es que el alcohol fue la droga más consumida (64\%). La prevalencia de consumo de drogas encontrada en este estudio fue semejante al Informe 2013 sobre alcohol, tabaco y drogas ilegales en España [22], en el que el mayor consumo entre las escolares españolas fue el alcohol, seguido por el tabaco y las drogas ilícitas.

Estudios hechos con muestras semejantes en España [6, 21, 23,] y en otros países [2, 24, 25], han hallado evidencias de un mayor consumo relacionado con el progresar de la edad. Tales resultados coinciden con los del presente estudio. Desde esta perspectiva, la edad puede ser considerada un factor de riesgo para el consumo, tanto de drogas lícitas como ilícitas, pues se vislumbra una mayor probabilidad de que las escolares consuman drogas al ir haciéndose mayores.

Quizás se pueda explicar porque el fumar y beber estén más presentes entre las escolares con mayor edad. Se supone que la libertad y autonomía conquistadas por tener una mayor edad y por existir una disminución de la supervisión de los padres, pueden llevar a una mayor independencia social y financiera, lo que facilitará el acceso a locales donde se venden más y se consumen más alcohol, tabaco, y otras drogas. Asimismo, puede haber una contribución del fenómeno "botellón" hoy presente en la rutina de algunas ciudades que se asocia, a menudo, con una ingesta muy grande de bebidas alcohólicas, cigarrillos y de otras drogas, [23].

Dando continuidad a los análisis, la Tabla 5 registra los datos respecto a la relación entre las drogas (lícitas e ilícitas) y el NAF de las escolares. 
Tabla 5: Consumo de drogas licitas (tabaco y alcohol) y drogas ilícitas en relación al nivel de actividad física de las escolares $(N=572)$

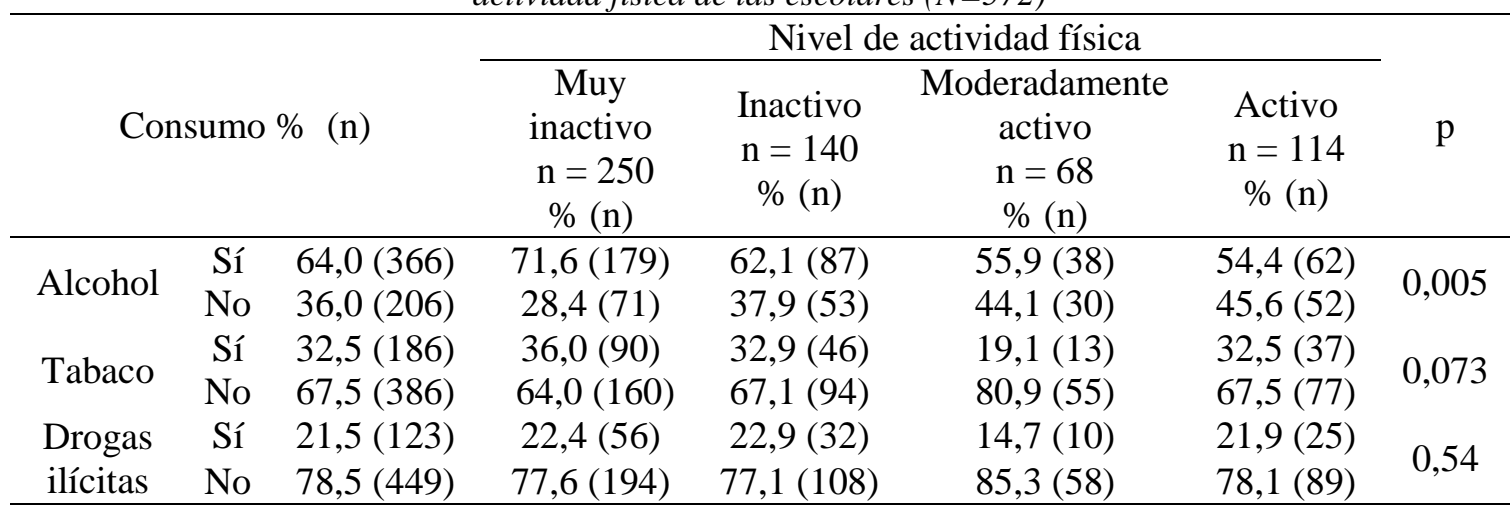

Los datos indicaron que el grado de actividad física se relacionó significativamente con el consumo de alcohol $\left(\chi^{2}=12,993 ; p=0,005\right)$, no así con el de tabaco $\left(\chi^{2}=6,953 ; p=0,073\right)$, ni con el consumo de drogas ilícitas $\left(\chi^{2}=2,145 ; \mathrm{p}=0,54\right)$. De hecho, tener un NAF activo provocó una disminución de la ingesta etílica. Parece que parte de los datos del presente estudio apoyarían la idea de que participar en actividades físicas y/o deportivas actúa como un factor protector respecto al uso de sustancias adictivas lícitas y/o ilícitas. No obstante, según Peck, Vida y Eccles (2008) [26], Peretti-Watel et al. (2003) [27], y Ruiz-Risueño (2010) [23], la relación entre esa práctica y la prevalencia del consumo de alcohol aun es imprecisa, siendo muy compleja de verificar. En esa misma línea, estudiosos que investigan la relación entre las actividades físico-deportivas y el consumo de drogas ilícitas no llegaron a informes concluyentes [28]. En el presente estudio, el NAF actuó como un factor protector hacia al consumo de sustancias adictivas entre las escolares, solamente para el consumo de alcohol.

Cuándo se analiza la prevalencia del consumo de bebidas alcohólicas respecto a la práctica de actividades físico-deportivas hay que tener cautela, pues se supone que dicha práctica depende de una serie de factores [29], como: los motivos, el tipo de actividad física, el tipo de deporte, el contexto, si es de participación o competitivo, si es colectivo o individual y, además, no se puede olvidar tener en cuenta la "hipótesis del deporte". De hecho, todos ellos podrán afectar los distintos resultados respecto a prevalencia del consumo etílico en relación a actividades físico-deportivas [30]. Para las comparaciones entre los resultados del presente estudio y otras investigaciones, se consideró la práctica de actividades físico-deportivas en general, independiente de su tipo, su intensidad y frecuencia, y de los posibles factores moduladores.

Desde esta perspectiva, se puede decir que las actividades físico-deportivas logran actuar como un factor preventivo hacia al consumo de bebidas alcohólicas, como fue demostrado en los estudios de Diehl et al. (2012) [4], Peretti-Watel et al. (2003) [27], Ruiz-Juan, y RuizRisueño (2010) [23], y Ruiz-Risueño et al. (2012) [25]. Todos ellos coinciden con los resultados de nuestro trabajo, lo que sugeriría que adoptar un NAF más o menos activo, podrá interferir en el etilismo de las escolares, lo que tal vez induzca a una menor prevalencia del consumo de bebidas alcohólicas. No obstante, algunos estudios han encontrado una mayor prevalencia del consumo de bebidas alcohólicas entre los practicantes de actividades físico-deportivas en relación a los no practicantes [24, 28, 29, 30, 31, 32] y Fredricks, y Eccles (2006) [33], no encontraron una asociación significativa entre actividades físico-deportivas y la prevalencia del consumo de alcohol y de drogas ilícitas en las escolares. Tales resultados no coinciden con los del presente estudio.

Parece que la naturaleza inquieta y exploradora del joven podría explicar la prevalencia del consumo de bebidas alcohólicas. Él joven está siempre buscando nuevas experiencias y actividades físico-deportivas, sea por necesidad de definir su identidad, socialización, aceptación social, y construir relaciones con sus pares o, solamente, para participar. De hecho, en los fines de semana ocurren reuniones sociales y de ocio, así como eventos deportivos que conllevan a un aumento del consumo etílico y también del tabaco. La explicación también 
puede estar en la "hipótesis del deporte" que supone celebraciones en el entorno de los deportes $\mathrm{y}$, principalmente, después de los eventos deportivos [31].

Peck et al. (2008) [26], indicaron que las prácticas físico-deportivas no actúan como un factor de riesgo o de protección hacia el consumo de alcohol entre los adultos jóvenes. Asimismo, parece que el etilismo sigue creciente entre los jóvenes y es influenciado por diversos factores personales y sociales y que van a actuar, de forma distinta, en cada persona, como la identidad y el temperamento del sujeto, sus retos, los motivos hacia la actividad, el entorno, sus pares, sus hábitos, el momento de su vida, además del tipo de deporte, el contexto no competitivo o competitivo, la intensidad y la cantidad de las sesiones de entrenamiento [30].

En el presente estudio, los hallazgos no apuntaron a una relación significativa al analizar la asociación entre los NAF y la prevalencia del tabaquismo y del consumo de drogas ilícitas entre las escolares, hechos similares a lo descrito por Rodríguez et al. (2013) [6]. Algunos estudios afirman la existencia de una relación inversa entre la práctica de actividad físico-deportiva y el consumo de tabaco y drogas ilícitas, pero hay que tener en cuenta las distintas metodologías y modelos estadísticos utilizados para los análisis de los datos, lo que podrá llevar a resultados diversos [21, 24, 25, 28, 32, 34]. Por el contrario, otras investigaciones han informado de una mayor prevalencia del consumo de esas drogas entre escolares practicantes de actividades físico-deportivas [27].

Tal vez los resultados contradictorios encontrados se deban a la compleja red que envuelve la investigación con seres humanos cuyas variables sociodemográficas, culturales y psicológicas son distintas en las muestras, así como la utilización de diferentes metodologías y de distintos aparatos de medida, cada una con sus clasificaciones específicas.

\section{CONCLUSIÓN}

En el presente estudio se pudo observar que el nivel de actividad física de escolares del sexo femenino se relacionó inversamente con la edad y con el consumo del alcohol. Respecto a la prevalencia de bebidas alcohólicas tener un buen nivel de actividad física actuó como un factor de protección. Nuestros datos apoyan estudios previos que apuntan a los beneficios de la práctica de actividades físico-deportivas para la salud física, mental y social, y la importancia de desarrollar dicho hábito en edades tempranas, con la esperanza de que se mantenga hasta la edad adulta. Es necesario el desarrollo en el ámbito escolar de programas pautados en ofertas de actividades físico-deportivas para los jóvenes, en particular para las chicas, conjuntamente con campañas educativas anti drogas. Los resultados a medio-largo plazo implicarían una reducción de costes en actividades de rehabilitación, disminuyendo, así, el gasto sanitario.

\section{REFERENCIAS}

1. Silva KSD, Nahas MV, Hoefelmann LP, Lopes ADS, Oliveira ESD. Associações entre atividade física, índice de massa corporal e comportamentos sedentários em adolescentes. Revista Brasileira de Epidemiologia. 2008; 11(1):159-168.

2. Farias Júnior JCD, Nahas MV, Barros MVGD, Loch MR, Oliveira ESAD, De Bem MFL, Lopes ADS. Comportamentos de risco à saúde em adolescentes no Sul do Brasil: prevalência e fatores associados. Revista Panamericana de Salud Pública. 2009; 25(4):344-352.

3. Hallal PC, Andersen LB, Bull C, Guthold R, Haskell W, Ekelund U, Lancet Physical Activity Series Working Group. Global physical activity levels: surveillance progress, pitfalls, and prospects. The Lancet. 2012; 380(9838):247-257.

4. Diehl K, Thiel A, Zipfel S, Mayer J, Litaker DG, Schneider S. How healthy is the behavior of young athletes? A systematic literature review and meta-analyses. Journal of Sports Science y Medicine. 2012; 11(2):201-220.

5. Pate RR, Stevens J, Webber LS, Dowda M, Murray DM, Young DR, Going S. Age-related change in physical activity in adolescent girls. Journal of Adolescent Health. 2009; 44(3):275-282. 
6. Rodríguez García PL, López Villalba FJ, López Miñarro PA, García Cantó E. Práctica de ejercicio físico y su relación con el consumo de tabaco en adolescentes. Diferencias entre géneros. Adicciones. 2013; (25)1:29-36.

7. Thomas JR; Nelson JK, Silverman S. Métodos de pesquisa em atividade física. $6^{\mathrm{a}}$ ed., Porto Alegre: Artmed; 2012.

8. Cantera-Garde MA. Niveles de actividad física en la adolescencia: estudio realizado en la población escolar de la provincia de Teruel [tesis doctoral]. Zaragoza (España): Facultad de Medicina Universidad de Zaragoza; 1997.

9. Cale L. Monitoring physical activity in children [tesis doctoral]. Loughborough (England): University of Technology at London; 1993.

10. Balaguer I. Un análisis de los factores determinantes de los estilos de vida saludables de los adolescentes valencianos de BUP, COU y FP. Dirección General d'Ensenyaments Universitaris i Investigació. Generalitat Valenciana (AE99-49); 1999.

11. Wold B. Health behaviour in schoolchildren: a WHO cross-national survey. Resource Package Questions 1993-1994. Norway: University of Bergen; 1995.

12. Beltrán-Carrillo VJ, Devís-Devís J, Peiró-Velert, C. Actividad física y sedentarismo en adolescentes de la Comunidad Valenciana. Revista Internacional de Medicina y Ciencias de la Actividad Física y el Deporte. 2012; 12(45):122-137. Disponible en <http://cdeporterediris.es/revista/revista45/artactividad266.htm >. Acceso en 12/02/2015.

13. Brooke HL, Corder K, Atkin AJ, van Sluijs EM. A systematic literature review with meta-analyses of within-and between-day differences in objectively measured physical activity in school-aged children. Sports Medicine. 2014; 44(10):1427-1438.

14. Garcinuño AC, García IP, Alonso IC, López SA. Determinantes del nivel de actividad física en escolares y adolescentes: estudio OPACA. Anales de Pediatría. 2011; 74(4):15-24.

15. Silva P, Aznar S, Aires L, Generelo E, Zaragoza J, Mota J. Differences in the physical activity pattern between Portuguese and Spanish adolescents. Archives of Exercise in Health and Disease. 2010; 1(1):26-31.

16. Treuth MS, Catellier DJ, Schmitz KH, Pate RR, Elder JP, McMurray RG, Webber L. Weekend and weekday patterns of physical activity in overweight and normal-weight adolescent girls. Obesity. 2007; 15(7):1782-1788.

17. Cantera-Garde MA, Devís-Devís J. Physical activity levels of secondary school Spanish adolescents. European Journal of Physical Education. 2000; 5(1):28-44.

18. Molinero O, Martínez R, Garatachea N, Márquez S. Pautas de actividad física de adolescentes españolas: diferencias mediadas por la participación deportiva y el día de la semana. Revista de Psicología del Deporte. 2010; (19)1:103-116, Disponible en <www.redalyc.org/articulo.oa?id=235116414007>. Acceso en 15/05/2015.

19. Peiró-Velert C, Devís-Devís J, Beltrán-Carrillo VJ, Fox KR. Variability of Spanish adolescents' physical activity patterns by seasonality, day of the week and demographic factors. European Journal of Sport Science. 2008; 8(3):163-171.

20. Gavarry O, Giacomoni M, Bernard T, Seymat M, Falgairette G. Habitual physical activity in children and adolescents during school and free days. Medicine and Science in Sports and Exercise. 2003; 35(3):525-531.

21. Tercedor P, Martín-Matillas M, Chillón P, Pérez López IJ, Ortega FB, Wärnberg J, Delgado M. Incremento del consumo de tabaco y disminución del nivel de práctica de actividad física en adolescentes españoles: estudio AVENA. Nutrición Hospitalaria. 2007; 22(1):89-94.

22. Observatorio Español de la Droga y las Toxicomanías (OEDT). Informe 2013: alcohol, tabaco y drogas ilegales en España. Madrid: Ministerio de Sanidad, Servicios Sociales e Igualdad, Delegación el Gobierno para el Plan Nacional sobre Drogas. 2014. Disponible en <http://docplayer.es/891308Informe-2013-alcohol-tabaco-y-drogas-ilegales-en-espana-o-d-t.html>. Acceso en 30/04/2015.

23. Ruiz-Juan F, Ruiz Risueño J. Ingesta de alcohol y práctica de actividad físico-deportiva en jóvenes. Revista Internacional de Medicina y Ciencias de la Actividad Física y del Deporte. 2010; 10(38):302322. Disponible en <http://cdeporte.rediris.es/revista/revista38/artingesta158.htm>. Acceso en 09/06/2015.

24. Lisha NE, Crano WD, Delucchi KL. Participation in team sports and alcohol and marijuana use initiation trajectories. Journal of Drug Issues. 2014; 44(1):83-93.

25. Ruiz-Risueño J, Ruiz-Juan F, y Zamarripa Rivera JI. Alcohol y tabaco en adolescentes españoles y mexicanos y su relación con la actividad físico-deportiva y la familia. Revista Panamericana de Salud Pública. 2012; 31(3):211-220.

26. Peck SC, Vida M, Eccles JS. Adolescent pathways to adulthood drinking: sport activity involvement is not necessarily risky or protective. Addiction. 2008; 103(s1):69-83. 
27. Peretti-Watel P, Guagliardo V, Verger P, Pruvost J, Mignon P, Obadia Y. Sporting activity and drug use: alcohol, cigarette and cannabis use among elite student athletes. Addiction. 2003; 98(9):12491256.

28. Lisha NE, Sussman S. Relationship of high school and college sports participation with alcohol, tobacco, and illicit drug use: a review. Addictive Behaviors. 2010; 35(5):399-407.

29. Mays D, DePadilla L, Thompson NJ, Kushner HI, Windle, M. Sports participation and problem alcohol use: a multi-wave national sample of adolescents. American Journal of Preventive Medicine. 2010; 38(5):491-498.

30. Lorente FO, Souville M, Griffet J, Grélot L. Participation in sports and alcohol consumption among French adolescents. Addictive Behaviors. 2004; 29(5):941-946.

31. Ford JA. Substance use among college athletes: a comparison based on sport/team affiliation. Journal of American College Health. 2007; 55(6):367-373.

32. Wichstrøm T, Wichstrøm L. Does sports participation during adolescence prevent later alcohol, tobacco and cannabis use? Addiction. 2009; 104(1):138-149.

33. Fredricks JA, Eccles J. Is extracurricular participation associated with beneficial outcomes? Concurrent and longitudinal relations. Developmental Psychology. 2006; 42(4):698-713.

34. Taliaferro LA, Rienzo BA, Donovan KA. Relationships between youth sport participation and selected health risk behaviors from 1999 to 2007. Journal of School Health. 2010; 80(8):399-410. 